\title{
Development of a Cryo-Air Protection Holder for FIB/(S)TEM
}

\author{
Y. Kuroda*, M. Kudo*, A. Morikawa*, T. Sato*, T. Iwahori**, and T. Agemura** \\ * Hitachi High-Technologies Corp., 11-1, Ishikawa, Hitachinaka-shi, Ibaraki, 312-0057, Japan \\ ** Hitachi High-Technologies Corp., 882, Ichige, Hitachinaka-shi, Ibaraki, 312-8504, Japan
}

The use of transmission electron microscopy (TEM) and electron energy loss spectroscopy (EELS) for the structural characterization and chemical analysis of materials for Lithium Ion Battery (LIB) applications is a growing focus area for fuel cell researchers. However, the high reactivity of Li metal ions found in LIB materials make it necessary to investigate these samples in environments that are free of oxygen to preserve the integrity of the samples. The samples must be maintained in inert environments when preparing or transporting the samples for microscopic analysis. In order to meet these stringent requirements, we have developed a fully compatible air protection specimen holder for FIB-STEM, which makes it possible to transport a specimen under an inactive gas condition [1].

Another area of development for FIB-STEM specimen holders centers on controlling the temperature of the specimen during observation. For certain types of materials fine structural changes can occur during FIB processing due to the interaction between ion beam and specimen. These fine structural changes are artifacts that are created by FIB fabrication and interfere with accurate structural analysis of the specimen. cryo-FIB milling techniques are known to be efficient method to reduce these artifacts during milling [2]. Here, we report on our recent progress in the development of a cryo-air protection FIB-STEM compatible specimen holder that provides both thermal and environmental protection for sensitive LIB materials.

FIG. 1 shows the FIB/STEM analysis system available to control atmosphere. The glove box used for preparing the specimens was filled with Ar gas, in which the humidity was kept the dew point of equal or less than $-30^{\circ} \mathrm{C}$ (FIG.1a). Here, the protective packaging was removed from the sample. The specimen was fixed onto the air protection holder and the protect cap was affixed to the end of the specimen holder (FIG.1b). The air-protection holder for bulk sample is used for the sample transportation in an Ar atmosphere from the glove box to the NB5000 FIB-SEM (FIG.1c). The specimen was set into the FIB-SEM and cooled to $-100^{\circ} \mathrm{C}$. A small section of the specimen was created by using the FIB micro-sampling technique on the cryo-air protection holder (FIG.1d) [3]. The specimen was thinned to about $100 \mathrm{~nm}$ thick using FIB milling voltage of $40 \mathrm{kV}$. Then, the STEM observation and EELS analysis were carried out by a Hitachi HD-2700 dedicated STEM with a cold field emission gun (FIG.1e) [4].

FIG. 2 provides an external view of the cryo-air protection compatible holder with STEM and FIB. The air protection cylinder slides over the tip of the specimen holder to protect the sample outside of the vacuum chamber or the inert atmosphere glove box that is used for sample preparation .

In this study, the cryo-air protection holder was used in conjunction with the FIB-SEM and HD-2700 STEM to analyze the environmentally sensitive LIB samples. 


\section{References}

[1] T. Sato, et al., Microscopy \& Microanalysis. M\&M, 212-213 (2010)

[2] M Marko, et al., Proc. Microscopy \& Microanalysis, 178-179 (2010)

[3] T. Ohnishi, and T. Ishitani, JP Patent 2774884 and US patent 5270552

[4] H. Inada, L. Wu, J. Wall, D. Su, \& Y. Zhu, J. of Electron Microsc. 58, 111-122 (2009)

(b) External view of air protection specimen holder for bulk specimen.

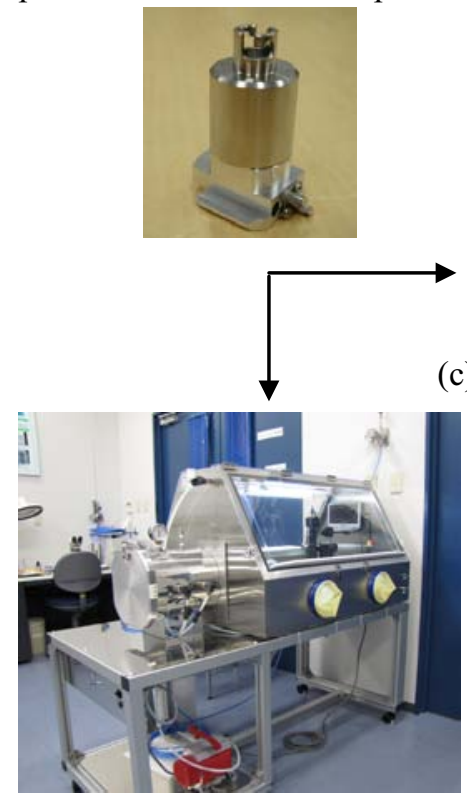

(a) External view of glove box. (d) External view of cryo-air protection FIB-STEM compatible specimen holder.
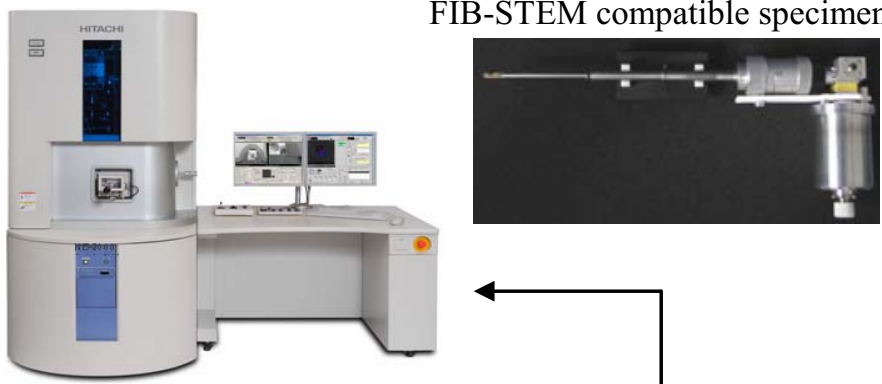

(c) A general view of NB5000 FIB-SEM.

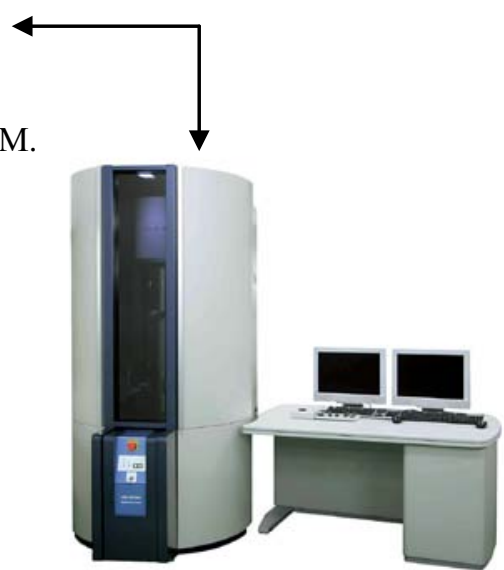

(e) A general view of HD-2700 STEM.

FIG.1 FIB/STEM analysis system used air protection holder.

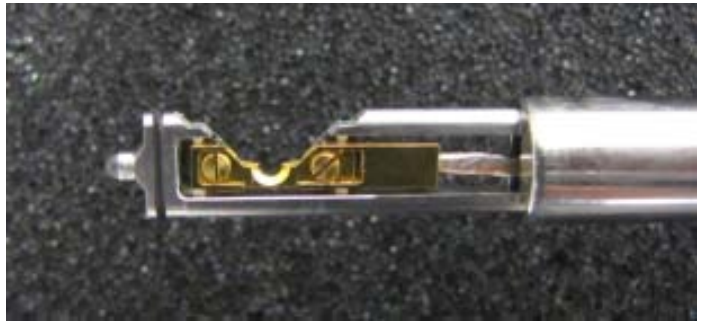

(a) Milling / Observation

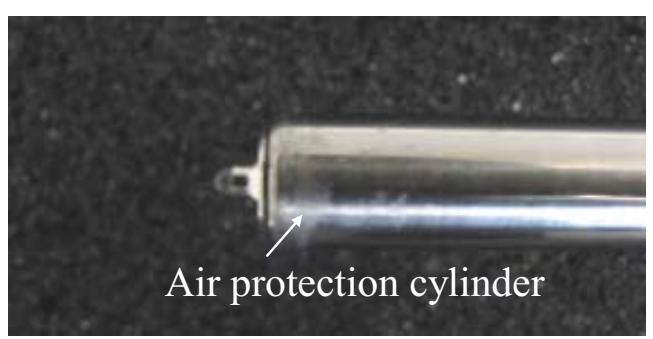

(b) Air protection

FIG.2 External view of cryo-air protection FIB-STEM compatible specimen holder of tip parts. 